\title{
Arquitectura inclusiva: un abordaje neurocognitivo
}

\author{
Inclusive architecture: a neurocognitive approach
}

\section{Resumen}

Autora:

Eska Elena Solano-Meneses* eskasolano@gmail.com

*Facultad de Arquitectura y Diseño

Universidad Autónoma del Estado de México

México

Recibido: 07/Jun/2020 Aceptado: 25/Oct/2020

- a arquitectura inclusiva escasamente se ha considerado bajo el sustrato de las ciencias neurocognitivas. Generalmente este enfoque de diseño arquitectónico se genera bajo lineamientos funcionales que no consideran niveles más profundos, como los neurocognitivos. Es por esta razón que el objetivo de este trabajo es analizar las implicaciones de las ciencias cognitivas en el diseño arquitectónico, para proponer pautas de diseño que contribuyan a fortalecer la inclusión en los espacios a través de códigos intuitivos. Para este fin, se apoya en un método inductivo, que inicia por una revisión analítica orientada a la arquitectura inclusiva, para contrastar con las propuestas desde las ciencias neurocognitivas, la accesibilidad, los principios de wayfinding y wayshowing y sus fundamentos semióticos. Se concluye que, en el diseño de espacios arquitectónicos, se hace necesario considerar el aprendizaje espacial y el pensamiento a través de patrones, ya que, como se ve, no es lo mismo que el aprendizaje visual y el pensamiento.

Palabras clave: arquitectura inclusiva; ciencias neurocognitivas; wayfinding; wayshowing.

\section{Abstract:}

Inclusive architecture has hardly been considered under the substrate of neurocognitive sciences. Generally, this approach to architectural design is generated under functional guidelines that do not consider deeper levels such as neurocognitive ones. It is for this reason that the objective of this work is to analyze the implications of cognitive sciences in architectural design in order to propose design guidelines that contribute to strengthening inclusion in spaces through intuitive codes. To this end, it relies on an inductive method, which begins with an analytical review oriented to inclusive architecture, to contrast with the proposals from the neurocognitive sciences, accessibility, the principles of wayfinding and wayshowing and their semiotic foundations. It is concluded that, in the design of architectural spaces, it is necessary to consider spatial learning and thinking through patterns, since as seen, it is not the same as visual learning and thinking.

Keywords: inclusive architecture; neurocognitive sciences; wayfinding; wayshowing. 


\section{Introducción}

Los avances en las ciencias neurocognitivas han desvelado una función mental inexplorada en el ser humano: la capacidad de orientación a través de la localización de puntos de interés, similar a un sistema de posicionamiento global mental que, convertidos en hábitos, facilitan el desplazamiento en un espacio o la manipulación de un objeto. Hoy sabemos que los sistemas de posicionamiento son generados en el hipocampo, mientras que los hábitos se generan en el cuerpo estriado del cerebro (Golfarb, 2016).

Estos avances de la ciencia posibilitan una nueva manera de abordar la arquitectura inclusiva desde las ciencias neurocognitivas, en lo que se ha denominado neuroarquitectura, la cual centra su enfoque en el entendimiento del entorno arquitectónico con relación a los procesos cerebrales de las personas (Green Building Council España, 2020). Es de este marco teórico que se desprenden conceptos como accesibilidad, wayfinding y wayshowing. Se entiende por accesibilidad al enfoque que privilegia la comprensión o entendimiento sencillo en el diseño. El concepto de Wayfinding considera los mecanismos cognitivos que, a través de la información que proporciona el entorno, forman parte del proceso de orientación del usuario en el espacio. Es decir, se centra en el sustrato. Por su lado, el wayshowing se relaciona con el dominio eficiente del proceso de orientación que implica percibir nuevos entornos partiendo de modelos prefigurados, a manera de modelos mentales, por lo que podemos afirmar que se centra en el proceso.

El objetivo de este trabajo es analizar las implicaciones de las ciencias cognitivas en el diseño arquitectónico para proponer pautas de diseño que contribuyan a fortalecer la inclusión en los espacios a través de códigos intuitivos. Se parte de la pregunta de investigación: ¿Cómo pueden contribuir los avances de las ciencias neurocognitivas en el diseño de una arquitectura inclusiva?, de donde deriva la formulación del supuesto que establece que las investigaciones de las ciencias neurocognitivas sobre percepción y orientación espacial permiten proponer principios de diseño que propicien un desplazamiento intuitivo y autónomo en cualquier espacio arquitectónico, dándole un carácter de inclusivo.

Se visualiza con ello la importancia quetienen dichos avances en el campo del diseño arquitectónico, especialmente en el inclusivo y para personas con discapacidad, porque implicaría una mirada profunda desde modelos mentales, y no a través de ajustes razonables que adaptan los espacios como una nueva forma de exclusión no universal. Estos estudios permiten delinear enfoques en el diseño arquitectónico inclusivo sustentados en recursos cognitivos innatos en los seres humanos.
Con el aprovechamiento de estos recursos es posible que los arquitectos diseñen considerando estos mapas mentales de ubicación para mejorar el desplazamiento a través del empleo consciente de códigos intuitivos (Aguilar Roblero, 2015). En ello radica la trascendencia de un enfoque neurocognitivo: mirar la arquitectura inclusiva no desde una óptica limitada a las normas de accesibilidad, sino desde conceptos emergentes, proveniente de las neurociencias cognitivas, así como de estrategias del wayfinding y el wayshowing.

Se considera que la arquitectura debiera aprovechar las investigaciones sobre percepción y orientación espacial, como una concepción no visual sino cognitiva, donde se exponga y explote la pertinencia de la capacidad mental de ubicación inherente al ser humano para que le sea posible optimizar desplazamientos en cualquier espacio. Si a estas posturas se suman los avances en diseño universal, la intención de lograr un desplazamiento autónomo para todos estaría más cerca de lograrse en aras de un espacio equitativo e inclusivo, que es un derecho de las personas señalado en la Convención sobre los derechos de las personas con discapacidad del 2006.

\section{Método}

De manera sintética, este trabajo se apoya en un método inductivo, que inicia por una revisión analítica orientada a la arquitectura inclusiva partiendo de los fundamentos de la teoría del diseño, para contrastar con las propuestas desde las ciencias neurocognitivas, la accesibilidad, los principios de wayfinding y wayshowing y sus fundamentos semióticos. Se establecen, asimismo, patrones delineados por las normas establecidas en la Convención sobre los derechos de las personas con discapacidad, celebrada en el año 2006, para proceder a generalizaciones que se concretan en una propuesta de Principios de diseño arquitectónico inclusivo bajo el concepto de wayfinding y wayshowing.

A manera descriptiva, las etapas de dicho trabajo son:

a. El análisis desde la teoría del diseño estudiando la relación que existe entre el diseño universal y la arquitectura inclusiva, centrándose en los enfoques sobre accesibilidad que les pueden vincular.

b. El análisis de los conceptos desde las ciencias neurocognitivas, encontrando que la interconexión de los conceptos de arquitectura inclusiva y wayfinding - wayshowing permiten no restringirse solo a cuestiones formales y funcionales en su diseño, sino abordar también los desplazamientos bajo un enfoque cognitivo, comunicativo y en consecuencia semiótico

c. El análisis de una mirada semiótica del wayfinding y del wayshowing, dado que desde la semiótica de Jakobson (1981) se podrán distinguir los elementos presentes en el proceso de comunicación: el diseñador (emisor), la persona (receptor), el espacio arquitectónico o urbano (canal) y las señales provenientes del wayfinding (el mensaje y código intuitivo).

d. Una evaluación del estado del arte, donde se muestra en los resultados la falta de incorporación de principios cognitivos e inclusivos en el diseño de espacios en la actualidad, señalando la necesaria 
revisión de las normas que demandan estos criterios con fundamento en los derechos humanos (ONU, 2008) como una manera de justificar la necesidad de esta mirada inclusiva en el diseño arquitectónico

e. Finalmente se propone una transferencia de los principios del wayfinding y del wayshowing al campo de la arquitectura para proponer pautas o principios de diseño con la finalidad de sistematizarlos y promover una arquitectura inclusiva.

\section{Marco Teórico}

\section{A. Arquitectura Inclusiva, diseño universal y ciencias neurocognitivas}

El diseño universal promueve un diseño que sea conveniente, accesible y comprensible para todo tipo de usuario, independientemente de sus particularidades, por lo que intenta eliminar adaptaciones o soluciones especializadas, que siempre terminan segregando a algún sector de la población. Acorde a Sanford (2016), para hacer un espacio accesible, el diseño universa se tendría que enfocar en aspectos específicos de la usabilidad, en vez de hacer adecuaciones. Su concepto trasciende el de accesibilidad de las personas con discapacidad para concebir una idea donde la inclusión, en todas sus manifestaciones, forme parte de las políticas urbanas en general. La importancia de esta perspectiva de diseño es que se ocupa de atender las necesidades de los usuarios más allá de los que se consideran promedio, típico o normal.

La arquitectura inclusiva es la respuesta de esta disciplina al diseño universal. Bajo este paradigma, su enfoque atrapa y rebasa al diseño sin barreras. La arquitectura inclusiva ha de considerar el rediseño de espacios con criterios de accesibilidad que satisfagan las necesidades de un usuario diverso, no como una concesión para alguno en particular, sino como una manera de englobarlos a todos.

En este sentido, la arquitectura inclusiva se apoya de las ciencias neurocognitivas y de la neuroarquitectura, las cuales parten de los estudios de procesos cerebrales y del descubrimiento de las células del lugar de O’Keefe y John Dostrovski. Esos científicos descubrieron que existían neuronas en una región concreta del hipocampo denominada CA1, que marcaban puntos de referencia que podían adaptarse a diferentes espacios (JiménezBalado, 2019)

De estos avances se desprenden diversos paradigmas. Por un lado, Arbib (2003) abunda en el estudio de los procesos neurocognitivos desde un enfoque de la neuroetología, para intentar comprender el comportamiento de los seres vivos partiendo de las funciones cerebrales, que posteriormente él usa para e desarrollo en paralelo de tecnologías en computadoras. Otra autora que se acerca a la idea de la arquitectura inclusiva es Berta Brusilovsky (2015). Sin embargo, ella lo hace desde un paradigma que entiende la orientación como una función neurológica hacia un diseño espacial que se suma a la de direccionamiento y comprensión de espacio con un enfoque hacia la accesibilidad cognitiva y no dependiente de manera específica de sistemas de información. Acorde a esta autora, la arquitectura es siempre un objeto funcional, en el que la misma función, la estructura, las relaciones espaciales y la estética deben expresarse como un texto legible, de lectura más intuitiva.

Desprendido de los avances de las ciencias neurocognitivas, el concepto wayfinding como estrategia de diseño fue primeramente introducido por Kevin Lynch (2015), cuya propuesta se inclina a la orientación y ubicación del usuario en la ciudad, entendiendo el wayfinding desde una capacidad cognitiva de orientación espacial y el wayshowing como manera de sistematizar su reforzamiento a través del diseño.

García Moreno (2012), define wayfinding como una estrategia apuntalada en una serie de mecanismos cognitivos que, a través de la información proporcionada por el entorno, apoyan el proceso de orientación del usuario en el espacio.

Para García Moreno (2012), el proceso de orientación está acotado por procedimientos perceptivos, cognitivos y de interacción; y no habla de recursos de orientación en sí mismos, sino de cómo se orientan las personas a través de procesos analíticos.

1. Procedimientos perceptivos: consisten en recursos de información obtenidos del entorno a través de canales auditivos, visuales y hápticos.

2. Procedimientos cognitivos: recursos de información obtenidos de la memoria almacenada y evaluada para la interacción con el espacio: el denominado esquema de ruta. El esquema de ruta se conforma con tres componentes: ubicación, destino y trayecto.

3. Procedimientos de interacción: la información que se va procesando obliga a las personas a actualizar la información de su entorno y posición, de modo que se ajuste la toma de decisiones en cada momento y lugar. Ello determina los recorridos en entornos arquitectónicos y urbanos.

Autores como Sussman y Hollander (2014) consideran la importancia de la relación que el cerebro mantiene con el ambiente, ya que aseguran que este genera respuestas inconscientes a los estímulos provenientes del entorno construido, los cuales determinan el comportamiento humano en nuestro medio ambiente, más allá de ser solo una respuesta estética y lógica a la percepción superficial, como se tenía entendido.

Con este enfoque de las ciencias neurocognitivas, autores como Taillade, N'Kaoua y Sauzéon (2016) han realizado algunos estudios sobre las relaciones entre la orientación y el comportamiento al desplazarse, analizando los niveles tácticos y estratégicos, sugiriendo una modificación en la manera de orientarse o desplazarse estrechamente ligada con la existencia de tareas específicas. A pesar de ello, aún no se ha establecido claramente la relación entre la manera de desplazarse y ubicarse en entornos urbanos con las estrategias de navegación preferidas por cada persona (Weisberg y Newcombe, 2016). 


\section{B. Arquitectura inclusiva y derechos de las personas con discapacidad}

A partir de la Convención sobre los derechos de las personas con discapacidad, celebrada en el año 2006 que pretende promover y proteger las condiciones de igualdad de las personas con discapacidad y el respeto de su dignidad, la sociedad se ha hecho más consciente de la situación de desequilibrio que este sector había enfrentado. Los espacios arquitectónicos, centrífugos y poco accesibles como consecuencia de su diseño, habían excluido en múltiples sentidos a minorías de la población, que por sus condiciones quedaban expulsadas de una vida plena. Acorde a De Paolis y Guerini (2015) resulta fundamental desarrollar códigos cuyas características permitan dar información de manera eficiente e inclusiva, considerando diferentes condiciones del usuario.

En el artículo 9 de la Convención citada, se establece que "para que las personas con discapacidad puedan vivir en forma independiente se adoptarán medidas pertinentes para asegurar el acceso en igualdad de condiciones al entorno físico, el transporte, la información y las comunicaciones e instalaciones abiertos al público o de uso público"(ONU, 2008, p.10). Con ello se abordan aspectos como la identificación y eliminación de obstáculos y barreras de acceso en los entornos urbanoarquitectónico.

Este documento se convierte en parteaguas a nivel mundial, y de manera paulatina los países comprometidos con dicha convención comienzan la implementación de medidas a través de normas que regulan las condiciones de los espacios, haciéndolos accesibles, aunque en muchos casos se manifiesta como lo preferible, pero no como algo obligado.

En México, para el año 2011 se promulga la Ley Genera para la Inclusión de las Personas con Discapacidad y en ella se establece que uno de los derechos fundamentales de las personas con discapacidad es la accesibilidad.

Las consideraciones a las normas evidentemente optimizan la función del espacio bajo el criterio de diseño universal; desafortunadamente no han considerado en sus principios los avances de las ciencias neurocognitivas, el diseño intuitivo bajo los principios del wayfinding y el wayshowing.

\section{Wayfinding}

Se entiende por wayfinding a la estrategia de diseño donde los sistemas de información apoyan a las personas para mejorar su comprensión y experiencia, tanto de objetos como del espacio arquitectónico. Para Golledge (1999), resulta imprescindible la comprensión a través de esta estrategia, de la manera en que los seres vivos pueden orientarse y utilizar la información espacial de una manera más eficiente para construir sistemas de orientación intuitivos.

Para ello considera la relación existente entre la diversidad (capacidades físicas, mentales, culturales, y sociales de las personas) y su relación con el ambiente en el que se desenvuelven. La importancia de una propuesta clara y legible del espacio urbano- arquitectónico resulta básica en situaciones de emergencia, pues ante una situación de peligro el cerebro sufre una desconexión racional y actúa por instinto, de manera que el desplazamiento para ponerse a salvo se debe de dar de modo intuitivo. El wayfinding propicia una postura activa del usuario, posibilitando tomas de decisiones con las que pueda ajustar su desplazamiento en situaciones imprevistas (Schwering, Krukar, Li, y Jo, 2017). Existen elementos comunicativos que los arquitectos han manejado y que culturalmente son utilizados para el reconocimiento del espacio. Ellos son: jerarquía, simetría, y zonificación funcional. Sin embargo, bajo el enfoque de la accesibilidad y del wayfinding, estos conceptos se traducen en principios que debieran ser vigilados de manera cercana para que, en conjunción con el diseño universal, se reduzca el riesgo para todos los usuarios.

Los principios del wayfinding, acorde a Mark A. Foltz $(1998$, p.59) son:

1. Crear una identidad en cada lugar diferente de todos los demás

2. Utilizar puntos de referencia para proporcionar señales de orientación y lugares memorables

3. Crear rutas bien estructuradas

4. Crear regiones, subdividiendo el espacio, con un carácter visual diferenciado

5. No dar a las personas demasiadas opciones en la navegación

6. Proporcionar mapas

7. Proporcionar señales en los puntos de toma de decisión para ayudar a tomar decisiones sobre el camino a seguir

8. Mostrar lo que se avecina, es decir, lo que nos vamos a encontrar a continuación

Estos principios, trasladados a la arquitectura inclusiva, resultan sumamente importantes en caso de emergencia, ya que de ellos depende el éxito de una ruta de evacuación o de la efectividad de las salidas de emergencia:

1. Crear una identidad a cada lugar, de manera que el usuario no se confunda. Los distintivos pueden ser colores, formas, texturas y aromas

2. Utilizar puntos de referencias como hitos, remates visuales, texturizados, etcétera

3. Crear rutas bien estructuradas, a través de composiciones de circulaciones lógicas, legibles, anchas y sin cambios de dimensiones, acusándose como ruta de evacuación

4. Subdividir el espacio, crear zonas o subzonas claramente identificables: las alturas y la iluminación constituyen señales perceptibles por personas con debilidad visual o ceguera (por el auxilio del sonido)

5. No dar demasiadas opciones de navegación - circulación, generando desplazamientos laberínticos o que no conducen a la salida

6. Colocar mapas de ubicación hápticos

7. Colocar señalamiento que indique salidas, que preferentemente sean mensajes y códigos arquitectónicos, como: cambio de altura, ampliar vanos, ampliar escalas, etcétera

8. Mostrar espacios contiguos y de preferencia espacios generales de ubicación (patios centrales que permitan entender la conformación global del espacio arquitectónico) 
Si a ello se suman las propuestas normativas con enfoque de accesibilidad y diseño universal, las posibilidades de que el espacio sea una barrera inaccesible se verán reducidas.

\section{Wayshowing}

Bajo la premisa de que el wayshowing posibilita el wayfinding, Per Mollerup (2013), diseñador y académico danés, reconfigura el papel pasivo del wayfinding -el qué- (como una estrategia orientada hacia la capacidad cognitiva que permite la ubicación y orientación espacial) para dirigir la mirada a las implementaciones espaciales que coadyuven al desarrollo de esta capacidad cognitiva: el wayshowing-el cómo-. Es decir, una vez comprendida la capacidad de los seres humanos de orientarse a través de la estrategia construida con códigos neurocognitivos de gran simpleza, el objetivo es perpetuar conscientemente dichos códigos en el diseño de los espacios.

El concepto del wayshowing, es un término acuñado por Per Mollerup (2013), entendido como un enfoque de diseño orientado a los sistemas cognitivos de información y orientación que optimizan la comprensión espacial. Mollerup (2013), desde esta trinchera, aboga por la simplicidad como base del diseño. Para él, la manera pertinente de mostrar el camino es reforzar una serie de códigos, ya inmersos en el cerebro humano, para sistematizarlos como un lenguaje que oriente al usuario en el espacio, independientemente de sus condiciones, y con ello propiciar espacios inclusivos.

Para Mollerup (2013) la base de este código recae en dos principios básicos en el diseño: simplicidad y redundancia. La simplicidad parte de una propuesta funcional donde el diseñador se apoya en los procedimientos perceptivos, cognitivos y de interacción, según los cuales son eliminados elementos que complicann los desplazamientos o configuraciones del lugar por considerar en su composición elementos que generan ruido, errores o malos entendidos. La redundancia, por su parte, constituye el eje de la sistematización de códigos, y en ello se sustenta la legibilidad del lugar.

Como se infiere, esto apoya la manera en que mentalmente se planea el desplazamiento por un lugar pero no como un símil a un mapa visual-mental, sino que involucra componentes simbólicos y experiencias pasadas que resultan determinantes en la ubicación espacial. La información que almacenamos en la memoria nos permite destacar aspectos del entorno necesarios o importantes para ubicarnos y desplazarnos en el espacio. Existe una interesante hipótesis, sustentada por Han y Ma (2015) que afirma que la manera en que almacenamos la información que usamos para significar los espacios o el entorno, se construye desde la infancia, y que es entonces cuando se definen aspectos de nuestra forma de desplazarnos y ubicarnos que pueden ser actitudinales y de percepción.

De la misma manera, es importante puntualizar que las investigaciones al respecto establecen que las condiciones de almacenamiento de información varían dependiendo de la dimensión del entorno urbano, ya que la complejidad del entorno también afecta el comportamiento de orientación en el espacio urbano (Li y Klippel, 2016). Aún aspectos como la densidad urbana y su relación con la legibilidad del entorno están en estudio.

Acorde al Centro de Diseño Inclusivo y Acceso Ambiental de la Universidad de Búfalo (2010), el diseño de los sistemas de orientación (wayshowing) debería considerar: a.- identificar y marcar espacios; b.- espacios de agrupamiento; c.- vincular y organizar espacios; y d. comunicar esta información al usuario.

A pesar de la conveniencia de adoptar medidas inclusivas y accesibles, la arquitectura actualmente se sigue rigiendo por medidas funcionalistas y racionalistas heredadas del movimiento moderno, donde el concepto de inclusión no existía.

Hoy día, los conceptos de zonificación que privilegian los arquitectos obedecen a principios funcionales de optimización de espacios (evitar desplazamientos y ahorrar espacios de construcción) y la relación entre ellos se da por lógica funcional y economía de instalaciones, pero no por conceptos de accesibilidad, wayfinding y wayshowing, que implican cuestiones de cognición espacial y de orientación con base a códigos y recursos simples.

Los espacios arquitectónicos deberían considerar el principio de un mapa cognitivo, es decir, la estructura cognitiva espacial, y con ello tres elementos:

1. Lugares (desde su concepto micro como mobiliario, hasta locales, patios, estructuras, hitos, etcétera)

2. Asociaciones entre lugares (considerar en el diseño la relación de distancia y dirección de un lugar con otro de manera que esta sea clara, legible y memorable)

3. Planes de desplazamiento o itinerarios (traducciones de los mapas cognitivos sobre el entorno).

Los elementos de construcción de los mapas cognitivos son las variables ambientales (Weisman, 1981), de las cuales se distinguen cuatro:

a. Acceso visual, generalmente manejado con elementos jerárquicos (como mayor escala, elementos arquitectónicos posicionadores como frontones, arcos, columnas gigantes, etcétera).

b. Grado de diferenciación, a través de distinciones en el manejo de los espacios generando contraste con color, forma, acabado y altura.

c. Complejidad del diseño espacial relacionado con la manera en que se presenta la articulación de los elementos espaciales entre sí.

d. Señalización, referido al uso o aplicación de recursos de orientación externos al individuo para facilitar los desplazamientos.

La falta de consideración de estos aspectos cognitivos hace que la mayor parte de los edificios carezcan de medidas que atiendan conceptos como los de la arquitectura inclusiva y el wayfinding - wayshowing de manera deliberada. 


\section{E. El wayfinding y el wayshowing desde una mirada semiótica}

Como se ha demostrado, uno de los principios básicos del diseño bajo el concepto de wayshowing es la simpleza y la eliminación de elementos que compliquen la legibilidad de los espacios. La posible legibilidad de un espacio se puede analizar semióticamente bajo dos criterios: la complejidad y la complicabilidad (Solano Meneses y Quiroga Llano, 2016).

La complejidad y la complicabilidad observan la función y el uso respectivamente. La función se entiende como una interpretación racional que es planteada por el emisor (diseñador), pero sujeta a las condiciones particulares del usuario. Por otro lado, el uso es pragmático por ser resultado de la interpretación del usuario y la manera en que se relaciona con el espacio. Este uso no necesariamente corresponde a la manera en que el autor lo concibió originalmente.

Se distinguen para su análisis los principios de la complejidad (que atiende al número de elementos y la función) y de la complicabilidad (que atiende a la dificultad que se pudiera presentar en el uso del espacio).

Los 4 principios de la complejidad que atienden a la función son:

1. Modelos conceptuales.- Residen en la memoria de usuario, son los esquemas mentales que permiten generar una estructura a priori de un espacio por experiencia previa, esto debido a la similitud entre elementos de un edificio con la misma condición esencial, facilitando la orientación y ubicación. Los emplazamientos y disposiciones van más allá de la estética, pues la lectura se puede apoyar en las características perceptuales, cognitivas y de interrelación que la persona conozca con anterioridad y resulte repetitiva.

2. Estructuras Modulares y Semas. - Este concepto se refiere a la conformación espacial de un edificio: al número de elementos que componen un edificio y su relación funcional. Este análisis estructural es resultado de la zonificación arquitectónica, que suele unir los diferentes espacios con base a sus funciones: zonas sociales, zonas íntimas y zonas de circulación, etcétera. Por ser un discurso frecuente en la arquitectura, puede servir como parte de los procedimientos cognitivos que el usuario ya porta con antelación.

3. Señales Gráficas. - Refiere a los signos que hacen legible un edificio. Se relaciona con la jerarquización de espacios que enfatiza aquellos de mayor demanda por su escala, tal es el caso de vestíbulos o accesos. En el lenguaje arquitectónico se expresan con: el tamaño, la forma, el color, los diferentes materiales empleados, etcétera. Estos recursos apoyan a la comprensión del espacio y a identificar su función.

4. Potencialidades. - Se refiere a la flexibilidad que puede tener un espacio, es decir, la capacidad de adaptarse a modificaciones, o de ser reutilizado de un modo distinto al original. Sin embargo, hay que considerar en estos cambios los códigos perceptuales y cognitivos ya descritos. Específicamente en la arquitectura latinoamericana, donde se privilegia la construcción permanente, esto es de gran valía.

Asimismo, los 4 principios de complicabilidad, que se refieren al modo en que los espacios son usados, son:

1. Ergonomía: Se pretende con ello un diseño que considere la optimización de recursos de desplazamientos y ubicación.

2. Affordances: Se apoyan en los códigos arquitectónicos que la gente comprende: por ejemplo, entender que las escaleras estarán cercanas a los elevadores, o que los vestíbulos posibilitan la distribución de las diferentes actividades en el edificio.

3. Protocolo y Secuencia de uso: Se refiere a una disposición lógica de los espacios obedeciendo una secuencia funcional. En una cocina se distinguen áreas como almacenaje, limpieza, preparación y cocción de los alimentos.

4. Tiempo de aprendizaje: El tiempo necesario para hacer posible la comprensión e identificación de los componentes en un edificio por parte de un usuario en su primera experiencia.

Las ideas de complejidad y complicabilidad funcionan como modelos de pensamiento, que remiten al intérprete o usuario a campos perceptivos, cognitivos y de interacción.

Estos contribuyen a la comprensión de los espacios, lo cual apuntala un enfoque semiótico que incide en las propuestas de accesibilidad, así como en las estrategias de wayfinding y de wayshowing ante sus principios comunicativos.

\section{Resultados: wayfinding y wayshowing como principios de la arquitectura inclusiva}

\section{Estado del arte en la incorporación de principios cognitivos e inclusivos en el diseño arquitectónico}

La falta de la inserción de conceptos de diseño desde las ciencias neurocognitivas ha generado una visión cerrada de un concepto funcional que busca la optimización y aprovechamiento de los espacios y no su disposición lógica, acorde a los procesos cognitivos, que facilite una arquitectura inclusiva.

Los problemas que genera la visión funcionalista, que se aleja de los principios del wayfinding y de la arquitectura inclusiva, es analizada en diferentes escenarios arquitectónicos, donde el proceso de evaluación deja evidencia de los errores que los arquitectos cometemos al ignorar los principios de arquitectura inclusiva, excluyendo a aquellos que por sus diversas condiciones debe lidiar con barreras. 


\begin{tabular}{|l|l|l|}
\hline $\begin{array}{l}\text { Problemas de di- } \\
\text { seño }\end{array}$ & \multicolumn{1}{|c|}{$\begin{array}{c}\text { Descripción de } \\
\text { errores comunes }\end{array}$} & Imagen \\
\hline $\begin{array}{l}\text { Obstaculizar la } \\
\text { libre circulación }\end{array}$ & $\begin{array}{l}\text { Los elementos se ubi- } \\
\text { can como islas impi- } \\
\text { diendo el libre despla- } \\
\text { zamiento }\end{array}$ & Imagen 1 \\
\hline $\begin{array}{l}\text { Disposición con- } \\
\text { fusa de espacios y } \\
\text { falta de jeraruiza- } \\
\text { ción de circual- } \\
\text { ciones }\end{array}$ & $\begin{array}{l}\text { Disposición confusa de } \\
\text { locales, sin jerarquiza- } \\
\text { ción ni simplificación de } \\
\text { circulaciones }\end{array}$ & Imagen 2 \\
\hline $\begin{array}{l}\text { Servicio poco } \\
\text { accesible }\end{array}$ & $\begin{array}{l}\text { Sanitarios en un nivel } \\
\text { diferente de una sala } \\
\text { de cines, en ubicación } \\
\text { poco visible e intuitivas }\end{array}$ & Imagen 3 \\
\hline $\begin{array}{l}\text { Servicios desvin- } \\
\text { culados }\end{array}$ & $\begin{array}{l}\text { Las escaleras tienen } \\
\text { que ser anunciadas con } \\
\text { un rótulo debido a la } \\
\text { poco pertinente solu- } \\
\text { ción arquitectónica }\end{array}$ & Imagen 4 \\
\hline $\begin{array}{l}\text { Circulaciones o } \\
\text { disposiciones con- } \\
\text { fusas y caprichosas }\end{array}$ & $\begin{array}{l}\text { Los sanitarios son solu- } \\
\text { cionados en condicio- } \\
\text { nes confusas y sin posi- } \\
\text { bilidad de asociación }\end{array}$ & Imagen 4 \\
\hline
\end{tabular}

Tabla 1: Problemas de diseño arquitectónicos derivados de la omisión de criterios inclusivos

Fuente: propia (2020)

En la figura 1 se aprecia un espacio que, si bien puede haber sido legible, los elementos se ubican como islas, impidiendo el libre desplazamiento al invadir áreas pensadas de circulación. El mismo problema de falta de legibilidad se aprecia en la figura 2, donde la disposición confusa de locales, sin jerarquización ni simplificación de circulaciones primarias, provoca espacios laberínticos. En el caso de la figura 3, se ubican sanitarios en un nivel diferente de una sala de cines, en ubicaciones poco visibles e intuitivas, además de ser un espacio inaccesible para personas con discapacidad motora, contraviniendo el principio de efecto agrupación y el de referencias e inferencias. En la figura 4, las escaleras, que resultan fundamentales para el desplazamiento, tienen que ser anunciadas con un rótulo (no accesible a personas con debilidad visual o con ceguera total) debido a la poco pertinente solución arquitectónica y a la ausencia de rampa, en contraposición al principio de efecto agrupación, apoyos de circulación vertical y al de rotura de efecto laberinto. Igualmente se incumplen los mismos principios en la figura 5 , donde los sanitarios son solucionados en condiciones laberínticas y sin posibilidad de asociación, además de la falta de una rampa.

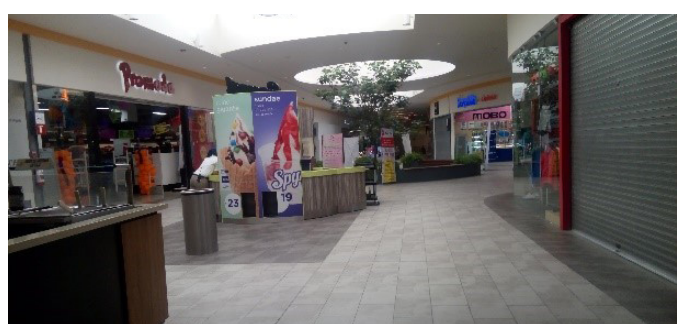

Figura 1: Instalaciones que interfieren el libre tránsito intuitivo y accesible

Fuente: propia (2017)

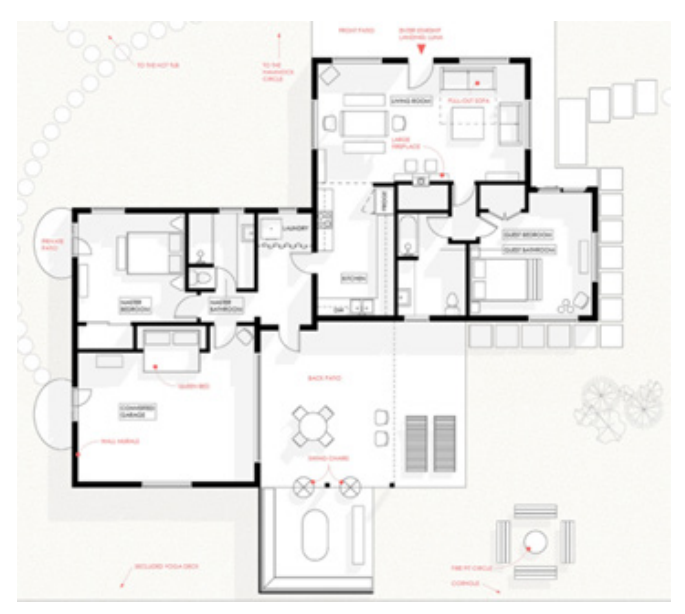

Figura 2: Disposición confusa de locales, sin jerarquización ni simplificación de circulaciones primarias, espacios laberínticos

Fuente:

https://images.adsttc.com/media/ $\mathrm{images} / 5 \mathrm{ca} 6 / 130 \mathrm{c} / 284 \mathrm{~d} / \mathrm{d} 1 \mathrm{a} 3 / 1 \mathrm{e} 00 / 02 \mathrm{fb} /$ slideshow/2018.03.03_Starship_Landing_Luna_Plan. jpg?1554387716

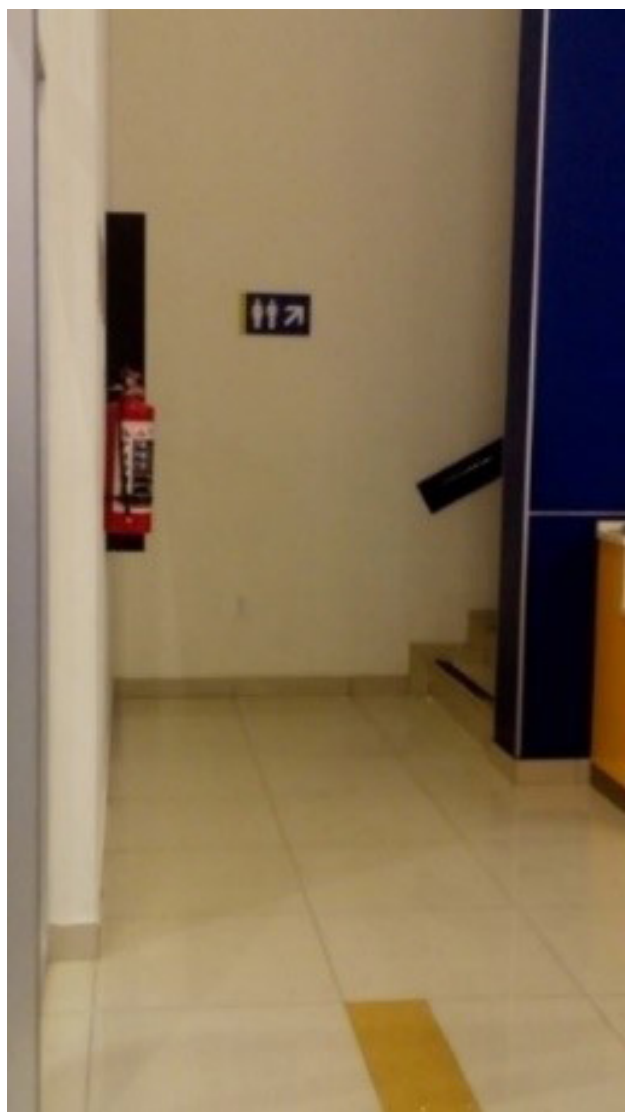

Figura 3: Sanitarios en un nivel diferente de una sala de cines, en ubicaciones poco visibles e intuitivas

Fuente: Propia (2017) 


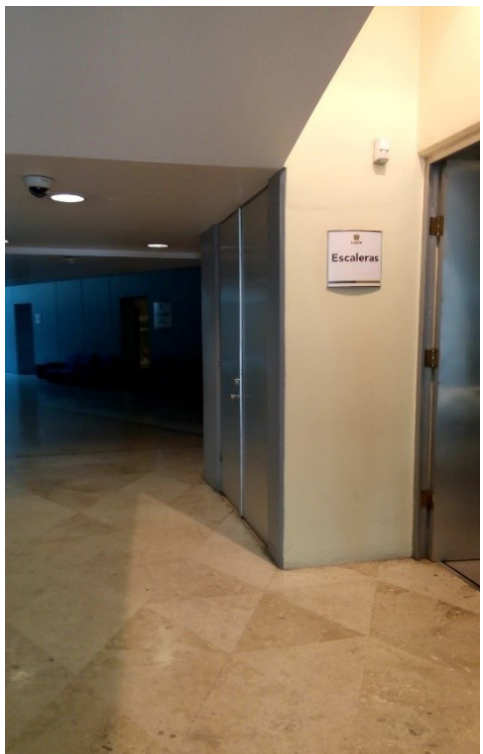

Figura 4: Las escaleras tienen que ser anunciadas con un rótulo debido a la poco pertinente solución arquitectónica

Fuente: propia (2017)

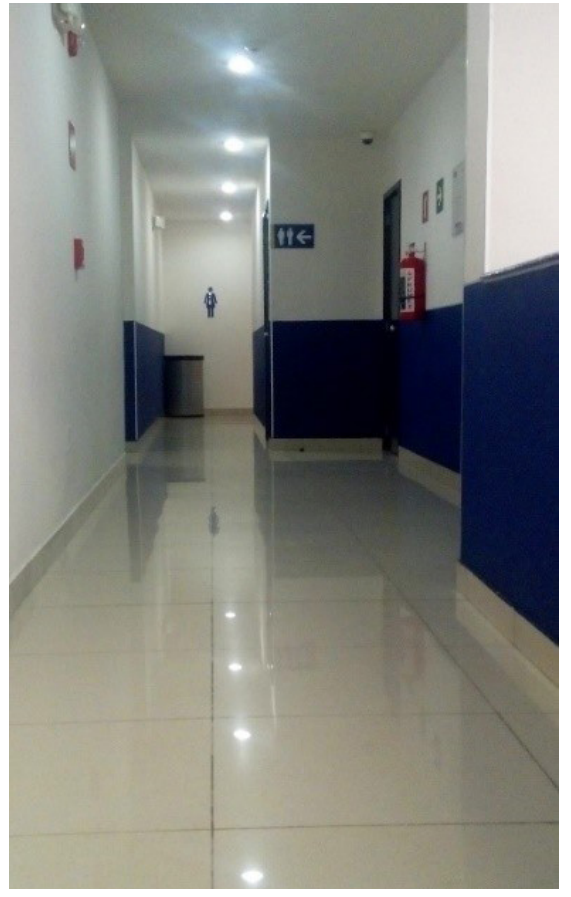

Figura 5: Los sanitarios son solucionados en condiciones confusas y sin posibilidad de asociación

Fuente: Propia (2017)

Todos estos elementos señalados constituyen barreras para los desplazamientos intuitivos, ya que no abonan a la construcción de un concepto de simpleza y redundancia para con ello contribuir con los procesos cognitivos en los que el instinto de orientación en el espacio resulta determinante. De ahí la importancia del concepto wayfinding y del wayshowing en el diseño arquitectónico inclusivo.

\section{Propuesta de principios de diseño arquitectónico inclusivo desde el wayfinding y el wayshowing}

Aunado a los diferentes paradigmas que nutren los fundamentos de una arquitectura inclusiva, se proponen, como producto de este trabajo, principios de diseño arquitectónico que, partiendo del wayfinding y del wayshowing, retoman y enriquecen las propuestas presentadas como antecedentes, y que buscan generar una disposición espacial y que se atiendan los siguientes aspectos ya discutidos. Estos principios, que constituyen una propuesta personal, pretenden conjuntar y abarcar principios provenientes de la semiótica, del diseño universal, de la accesibilidad universal, y sumar criterios cognitivos, para con ello establecer principios de diseño que conduzcan a la construcción de una arquitectura inclusiva. Estos son:

1. Normalización, generalización y sistematización de patrones funcionales (Riffing),

2. Visualización de elementos de orientación (hitos perceptuales)

3. Uso del color como código de desplazamiento $y$ ubicación,

4. Uso de guías olfativas y sensoriales,

5. Recorridos directos, rectos y sin barreras

6. Alturas de cubiertas y anchos de circulaciones que sirven de códigos de jerarquías

7. Remates como guías, elementos configurados como hitos

Los principios referidos se exponen como posibles líneas de intervención en el diseño, lo que supone un aterrizaje de las propuestas teóricas y estudios aledaños ya referidos, consolidados en una propuesta que busca amalgamar, pero, sobre todo, simplificar las propuestas de diseño:

1. Normalización, generalización y sistematización de patrones funcionales (Riffing), que si bien la zonificación de los espacios se ha dado por enfoques funcionales (atendiendo intereses económicos de eficiencia de instalaciones y desplazamientos), hoy se reconceptualiza no solo por economía de desplazamientos, sino por lógica cognitiva. A ello se puede abonar que espacios con funciones cercanas homologuen acabados, materiales y ubicaciones espaciales para crear un mapa cognitivo que obedezcan a la asociación por semejanza o similitud. De esta manera, espacios comunes compartirían acabados en pisos, muros y plafones, así como colores y materiales de preferencia con cualidades olfativas. La figura 6 muestra que una homologación en cuanto a la continuidad de los espacios (puertas continuas y alineadas) y materiales, alturas, etcétera, permiten una orientación intuitiva en lugares que obedecen a una misma función, y con ello podrían distinguirse de otros con funciones diferentes, donde un cambio de altura o material sea el código. 


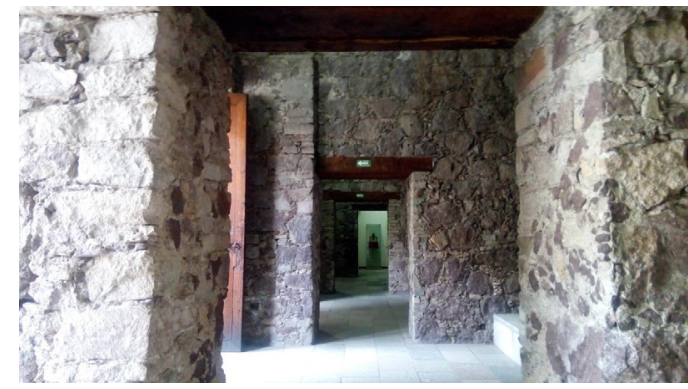

Figura 6: Homologación de alturas, acabados en muros y continuidad situacional como ejemplo de patrones funcionales

Fuente: propia (2017)

2. Visualización de elementos de orientación (hitos perceptuales). Implica ir dejando indicios o señales que, a través de su consecución, permitan al usuario entender el espacio de manera global y construir con ello su recorrido. Ello requiere de códigos que el diseñador proponga como: dobles alturas, manejo de domos, tratamientos en piso, etcétera; sobre todo en espacios públicos, que permitan visualizar los hitos independientemente de la disposición o emplazamiento de las diferentes zonas del edificio. Se propone concientizar a los diseñadores sobre la oportunidad de que códigos de orientación, como son los tratamientos en los pisos, se usen de manera útil y no estética, como en la figura 7.

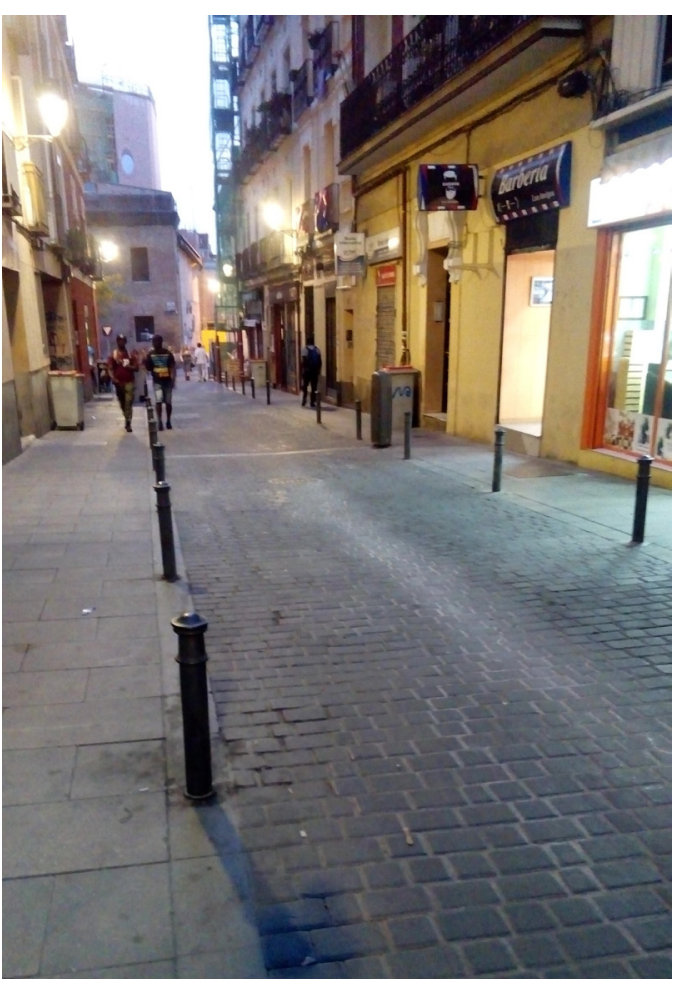

Figura 7: Ejemplo de una adecuada aplicación de diseño de acabado, texturas y colores en piso, que se sustenta en los conceptos de Wayshowing al aprovechar la posibilidad de definir el uso de cada espacio (peatonal y vehicular)

Fuente: propia (2019)
3. Uso del color como código de desplazamiento y ubicación, que constituye una señal que facilita la ubicación, en la cual el cambio de color indica cambio de zona en el espacio arquitectónico o urbano, o cambio de función espacial. En espacios confusos, como edificios de planta tipo (hoteles, estacionamientos, etcétera), el color es un argumento de ubicación. Los colores pueden apoyar a la identificación, por ejemplo, de diferentes salas en un museo, como sucede en el museo MARCO, en Monterrey, México, donde cada sala tiene diferente color (Figura 8).

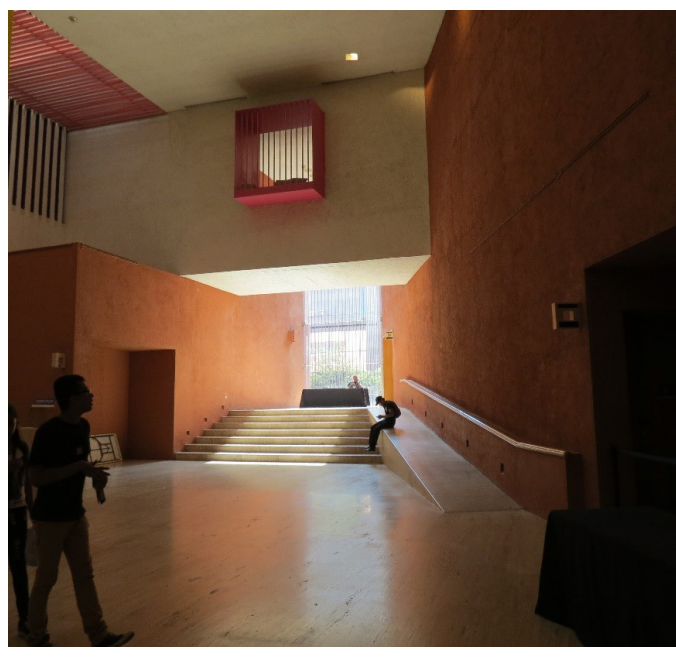

Figura 8: El manejo del color como recurso de ubicación en el museo MARCO; Monterrey, México Fuente: propia (2015)

4. Uso de guías olfativas y sensoriales, de manera que signos como la luz, los aromas y las corrientes de aire generados con recursos como patios interiores, jardines, pozos de luz, puedan ser elementos que faciliten la ubicación en el espacio, a la vez que cumplen como sistemas pasivos para aminorar demandas de energía e instalaciones no sustentables. De ahí la importancia de incorporar estos espacios que hoy cobran especial valor, ya que constituyen fuentes naturales de luz y ventilación, pero, además, ayudan al usuario a ubicarse en el espacio arquitectónico (Figura 9).

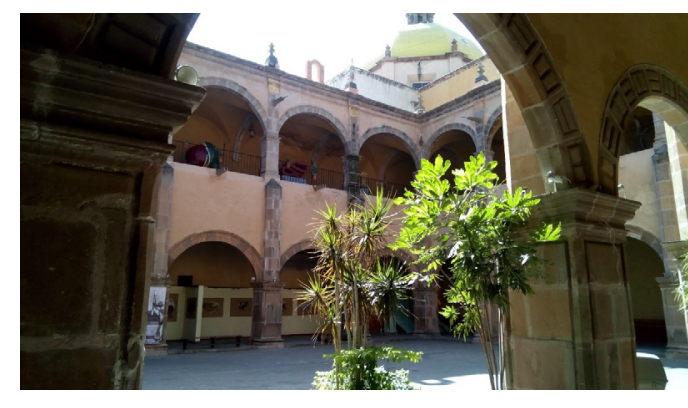

Figura 9: La recuperación de espacios como los claustros o espacios de doble altura, donde estén presentes espacios abiertos, corrientes de aire, plantas o jardines con aromas, y mayor iluminación, para construir con ello un referente espacial Fuente: propia (2017) 
5. Recorridos directos, rectos y sin barreras, con lo cual es necesario evitar elementos como columnas, mobiliario, plantas y demás elementos en medio de las circulaciones que constituyen una barrera o generan ruido en la construcción de mapa cognitivo del usuario. Los desplazamientos recomendablemente deben ser claros, rectos, libres de obstáculos y que muestren el destino, como se observa en la figura 10.

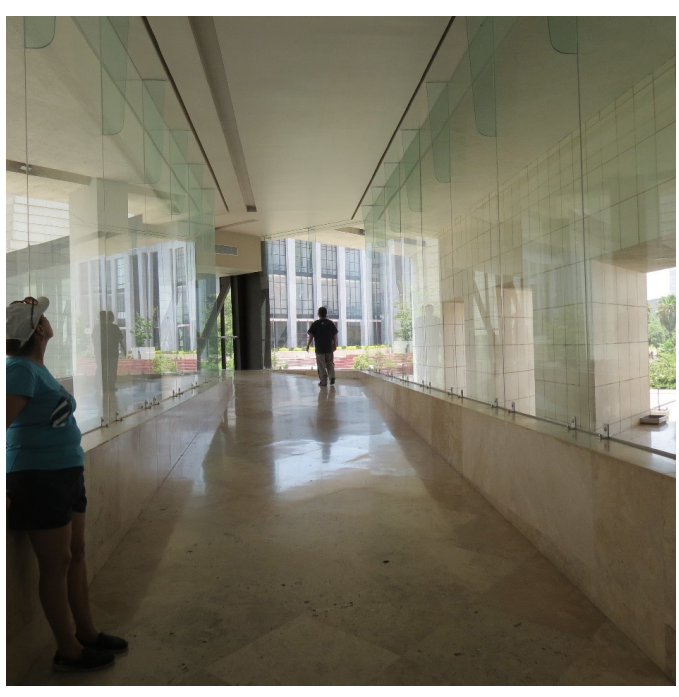

Figura 10: Ejemplo de desplazamiento libre de obstáculos, simple y recto en el MUNE, Monterrey Fuente: propia (2015)

6. Alturas de cubiertas y anchos de circulaciones que sirven de códigos de jerarquías, cuyo patrón reconoce que, a mayor ancho de circulación, mayor importancia del andador, pasillo o calle. Con ello es posible recurrir a los deambulatorios de mayor dimensión para localizar las salidas.

7. Remates como guías, elementos configurados como hitos, de los cuales se aprovechen sus cualidades sonoras, como el caso de fuentes o caídas de agua, pero siempre buscando evitar conflictos físicos por su ubicación y no confundir con propuestas estéticas banales. Esto señala la importancia de concebir estos recursos como códigos de ubicación inclusiva (para personas con discapacidad visual o con Alzheimer).

\section{Conclusiones}

La importancia de la integración de las ciencias neurocognitivas en el diseño arquitectónico, estriba en los beneficios que para todas las personas implica. La diversidad se resuelve en el espacio a través de una mejora en el diseño de espacios, accesos y circulaciones, promoviendo la arquitectura inclusiva y colocando a las personas con discapacidad en igualdad de derechos y con mejores condiciones de calidad de vida.

El concepto actual de discapacidad no está más sujeto a características intrínsecas de la persona, sino al contrario, está definido como el resultado de una compleja relación entre la condición de salud y los factores externos. Es por ello que es necesario ser conscientes de que los ambientes que diseñamos son determinantes para todos, pero en especial para las personas con discapacidad, ya que si presentan barreras de cualquier tipo dificultarán el funcionamiento y restringirán el desempeño de las personas con discapacidad, es decir, es el entorno el que discapacita e inhabilita.

Sin embargo, a pesar de la importancia que ya se ha discutido aquí, referente a los principios de la arquitectura inclusiva, su implementación en la construcción y la eficiencia operativa de los edificios, no existen normas reguladas sobre el diseño que unifiquen el concepto de Diseño Universal con los conceptos de wayfinding y wayshowing.

En el diseño de espacios arquitectónicos se hace necesario considerar el aprendizaje espacial y el pensamiento a través de patrones, ya que, como se ve, no es lo mismo que el aprendizaje visual y el pensamiento.

Integrar el concepto de wayfinding y el wayshowing en el diseño arquitectónico implica el uso de entradas sensoriales y cognitivas, generalmente no consideradas en las propuestas funcionales y estéticas que predominan en el diseño no inclusivo. Las cualidades de los signos arquitectónicos brindan la posibilidad de que, sustentados en la simplicidad y la redundancia, principios ya expuestos del wayshowing, se propicie la inclusión para personas con discapacidad, a través de una asociación más significativa con el espacio que se vive. Es por ello que, explorar los códigos y recursos arquitectónicos configurados con los procedimientos perceptivos, cognitivos y de interacción, permite la legitimación de los mismos como un medio de socialización que enriquezca la comprensión e inclusión de las personas a quienes un criterio arquitectónico funcionalista y estético les impide experimentar el mundo que les rodea.

Es compromiso de los arquitectos y diseñadores, construir enfocándose en los sistemas de orientación que incluyan la implementación de los principios de Diseño Inclusivo a través de la generación de espacios que contemplen los principios aquí descritos desde los conceptos de wayfinding y wayshowing: generar espacios de agrupamiento o zonificación sustentados en propuestas de normalización; generalización y sistematización de patrones funcionales; visualización de elementos de orientación; uso del color como código de desplazamiento y ubicación; uso de guías olfativas y sensoriales; recorridos directos, rectos y sin barreras; alturas de cubiertas y anchos de circulaciones y remates como guías; vincular y organizar espacios bajo esquemas cognitivos básicos permitiendo la identificación, simplificación y ordenamiento de aspectos que faciliten la ubicación (acceso visual, grado de diferenciación a través de contrastes de color, forma, acabado y altura) disminución de la complejidad del diseño espacial con una relación adecuada de los elementos espaciales entre sí y señalización conveniente; y, finalmente, comunicar esta información al usuario de manera legible y universal a través de la sistematización de estos procesos. 
Cómo citar este artículo/How to cite this article: Solano-Meneses, E. E. (2021). Arquitectura Inclusiva: un abordaje neurocognitivo. Estoa. Revista de la Facultad de Arquitectura y Urbanismo de la Universidad de Cuenca, 10(19), 103-113. doi: 10.18537/est.v010.n019.a09

\section{Referencias bibliográficas}

Aguilar Roblero, R. (2015). El sistema de posicionamiento cerebral: Premio Nobel en Fisiología y Medicina 2014 Revista de la Facultad de Medicina de la UNAM, 58 (3), 53-58. https://www.medigraphic.com/pdfs/facmed/un2015/un153i.pdf

Arbib, M. (2003). The Handbook of Brain Theory and Neural Networks. The MIT Press.

Brusilovsky, B. (2015). Accesibilidad Cognitiva Modelo para diseñar espacios accesibles. Ciudad Accesible de Granada.

Cámara de Diputados del H. Congreso de la Unión (2018). Ley General para la Inclusión de las Personas con Discapacidad. Diario Oficial de la Federación.

http://www.diputados.gob.mx/LeyesBiblio/pdf/ LGIPD_120718.pdf

De Paolis, R. y Guerini, S. (2015). Wayfinding Accessible Design. En C. Gambardella (Ed.), Heritage and Technology (pp. 1411-1420). La Scuola di Pitagora editrice.

Foltz, M. A. (1998). Designing Navigable Information Spaces [Tesis de Maestría, Massachusetts Institute of Technology]. http://rationale.csail.mit.edu/pubs/mfoltz/ mfoltz-thesis/thesis.pdf

García Moreno, D. (2012). Diseño de Sistemas de Orientación Espacial: Wayfinding. Laboratorio Wayfinding.

Golfarb, E. (2016). Memory guided attention: Independen contributions of the hippocampus and striatum. Neuron.

Golledge, R. (1999). Wayfinding Behavior, Cognitive Mapping and Other Spatial Processes. The Johns Hopkins University Press.

Green Building Council España. (2020). Salud, espacios, personas. HAUS. https://gbce.es/documentos/SaludEspacios-Personas.pdf

Han, S. y Ma, Y. (2015). A culture-behavior-brain loop model of human development. Trends in Cognitive Sciences, 19(11), 666-676.

Jakobson, R. (1981). Linguística, poética, tiempo. Crítica.

Jiménez-Balado, J. (2019). Las 'células de lugar', algo asi como nuestro GPS cerebral. Psicología y mente. https:// psicologiaymente.com/neurociencias/celulas-lugar-gpscerebral
Li, R. y Klippel, A. (2016). Wayfinding behaviors in complex buildings: The impact of environmental legibility and familiarity. Environment and Behavior, 48(3), 482-510.

Lynch, K. (2015). La imagen de la ciudad. Gustavo Gili.

Mollerup, P. (2013). Wayshowing $>$ Wayfinding Basic and Interactive. BIS Publisher.

ONU. (2008). Convención sobre los derechos de las personas con discapacidad. ONU.

Sanford J.A. (2016). Design for All Users. En R. Hunter, L. Anderson y B. Belza (Eds.), Community Wayfinding: Pathways to Understanding (pp. 81-101). Springer

Schwering, A., Krukar, J., Li, R., Joy, V. y Fuest, S. (2017). Wayfinding Through Orientation. Spatial Cognition \& Computation, 17, 273-303. https://www.tandfonline. com/doi/full/10.1080/13875868.2017.1322597

Solano Meneses, E. y Quiroga Llano, C. (2016). Complejidad y complicabilidad en Arquitectura. Revista Ontosemiótica, 3 (8), 49-67.

Sussman, A. y Hollander, J. B. (2014). Cognitive Architecture: Designing for how we Respond to the Built Environment. Routledge.

Taillade, M., N'Kaoua, B. y Sauzéon, H. (2016). Agerelated differences and cognitive correlates of selfreported and direct navigation performance: The effect of real and virtual test conditions manipulation. Frontiers in Psychology, 6, 2034

Weisman, J. (1981). Evaluating architectural legibility: way-finding in the built environment. Environment and Behavior 13 (2), 189-204.

Weisberg, S. M. y Newcombe, N. S. (2016). How do (some) people make a cognitive map? Routes, places, and working memory. Journal of Experimental Psychology: Learning, Memory, and Cognition, 42(5), 768. 